\title{
The glutathione import system satisfies the Staphylococcus aureus nutrient sulfur requirement and promotes interspecies competition.
} C. Shook ${ }^{a}$, Phillip C. Delekta ${ }^{a}$, Christopher C. Cooper ${ }^{d}$, Daniel Havlichek Jr. ${ }^{d}$, Martha H. Mulks ${ }^{a}$, Sophia Y. Luntc, e, Janani Ravi ${ }^{b}$, \& Neal D. Hammer ${ }^{*}$

a Department of Microbiology and Molecular Genetics, Michigan State University, East Lansing,

${ }^{b}$ Department of Pathobiology and Diagnostic Investigation, Michigan State University, East

${ }^{\mathrm{C}}$ Department of Biochemistry and Molecular Biology Michigan State University, East Lansing, MI 17 48824, USA

${ }^{\mathrm{d}}$ Department of Medicine, Division of Infectious Disease, Michigan State University, East Lansing, MI 48824, USA

e Department of Chemical Engineering and Materials Science, Michigan State University, East

${ }^{*}$ Corresponding author hammern2@msu.edu 


\section{ABSTRACT}

Sulfur is an indispensable element for proliferation of bacterial pathogens. Prior studies

32 indicated that the human pathogen, Staphylococcus aureus utilizes glutathione (GSH) as a source

33 of nutrient sulfur; however, mechanisms of GSH acquisition are not defined. Here, we identify a

34 previously uncharacterized five-gene locus comprising a putative ABC-transporter and y-glutamyl

35 transpeptidase (ggt) that promotes $S$. aureus proliferation in medium supplemented with either

36 reduced or oxidized GSH (GSSG) as the sole source of nutrient sulfur. Based on these

37 phenotypes, we name this transporter the Glutathione import system (GisABCD). We confirm that

38 Ggt is capable of cleaving GSH and GSSG $\mathrm{Y}$-bonds and that this process is required for their use

39 as nutrient sulfur sources. Additionally, we find that the enzyme is cell associated. Bioinformatic

40 analyses reveal that only Staphylococcus species closely related to $S$. aureus encode GisABCD-

41 Ggt homologues. Homologues are not detected in Staphylococcus epidermidis. Consequently,

42 we establish that GisABCD-Ggt provides a competitive advantage for $S$. aureus over $S$.

43 epidermidis in a GSH-dependent manner. Overall, this study describes the discovery of a nutrient

44 sulfur acquisition system in $S$. aureus that targets GSH and promotes competition against other

45 staphylococci commonly associated with the human microbiota. 
Bacterial pathogens are limited to metabolites present in host tissues to fulfill nutritional requirements during infection. Strategies pathogens employ to acquire nutrient transition metals,

50 such as iron, are well established ${ }^{1,2}$. However, studies defining mechanisms that support nutrient

51 sulfur acquisition have been restricted to a limited number of pathogens ${ }^{3}$. Sulfur is essential due

52 to its capacity to fluctuate between redox states and therefore catalyzes numerous cellular

53 reactions ${ }^{4,5}$. Ultimately, cells require sulfur to synthesize cysteine (Cys). Cys is the fulcrum of

54 sulfur metabolism as an intermediate for methionine (Met) and cofactors such as Fe-S clusters ${ }^{4,6-}$

558 . In host cells and some bacterial species, Cys is also required to generate the low molecular

56 weight thiol glutathione $(\mathrm{GSH})^{9}$.

GSH concentrations range between 0.5 and $10 \mathrm{mM}$ in mammalian tissues, making it a

58 relatively abundant source of nutrient sulfur for invading pathogens ${ }^{9-20}$. In addition to Cys, GSH

59 consists of glutamate and glycine. A unique $\mathrm{Y}$-bond links the glutamate $\mathrm{Y}$-carboxyl to the Cys

60 amine. To maintain Cys reservoirs, organisms rely on GSH catabolism via the $\mathrm{y}$-glutamyl cycle ${ }^{9,21}$.

61 Liberation of Cys from GSH is a two-step process that requires $\mathrm{Y}$-glutamyl transpeptidase (Ggt),

62 a specialized peptidase conserved in all domains of life due to its role in the $\mathrm{y}$-glutamyl cycle $\mathrm{e}^{9,22-}$

$63{ }^{25}$. Typically, Ggt is localized to the periphery of the cell where it degrades endogenous GSH in

64 the extracellular milieu or within the Gram-negative periplasm ${ }^{26-28}$. Ggt has been implicated in

65 pathogen nutrient sulfur acquisition via Cys liberation in only one species, Francisella tularensis ${ }^{18}$.

67 in the United States and Europe ${ }^{29-31}$. Strategies S. aureus employs to obtain nutrient sulfur during

68 pathogenesis are largely unknown. Moreover, while GSH is an established in vitro source of

69 nutrient sulfur for $S$. aureus, mechanisms of GSH acquisition have not been defined ${ }^{32}$. S. aureus

70 does not synthesize GSH but encodes a putative Ggt. This fact supports the hypothesis that

71 staphylococcal Ggt catabolizes exogenous host GSH, liberating Cys as a means to satisfy the

72 nutrient sulfur requirement ${ }^{33}$. Here we demonstrate that $S$. aureus utilizes oxidized GSH (GSSG)

73 as a nutrient sulfur source and isolate mutants that fail to proliferate in medium supplemented 
74 with GSSG or GSH as the sole source of nutrient sulfur. These mutants harbored transposon (Tn)

75 insertions within a five gene locus, SAUSA300_0200-0204. SAUSA300_0200-0203 encodes a 76 predicted ATP-binding-cassette (ABC) transporter and SAUSA300_0204 encodes a putative Ggt.

77 Consequently, we name this transporter the Glutathione import system (GisABCD). We

78 determine that $S$. aureus Ggt associates with the cell and that the recombinant enzyme cleaves

79 both GSH and GSSG. A query for GisABCD-Ggt across Firmicutes revealed that only a select

80 clade within the Staphylococcus genus, one that does not include S. epidermidis, encodes

81 homologues of the system. Consistent with this, S. aureus outcompetes S. epidermidis in GSSG-

82 or GSH-supplemented conditions. Therefore, this newly described nutrient sulfur acquisition

83 system provides a competitive advantage for $S$. aureus over other staphylococci commonly

84 associated with the human microbiota.

Results

87 S. aureus proliferates in medium supplemented with GSSG as the sole source of nutrient

88 sulfur. A previous study qualitatively reported that $S$. aureus generates colonies on a chemically-

89 defined medium supplemented with GSH as the sole sulfur source, indicating that the abundant

90 host metabolite is a viable source of nutrient sulfur ${ }^{32}$. However, $S$. aureus likely encounters both

91 reduced and oxidized GSH (GSSG) as the pathogen induces a potent oxidative burst during

92 infection ${ }^{34}$. Therefore, we hypothesized that $S$. aureus utilizes GSSG as a source of nutrient sulfur.

93 To quantitatively assess utilization of GSH and GSSG as sulfur sources by $S$. aureus, a 94 chemically-defined medium, referred to as PN, was employed ${ }^{35}$. PN contains sulfate and 95 methionine (Met), but $S$. aureus lacks the capacity to assimilate sulfate or utilize Met as sources 96 of sulfur; thus, cystine (oxidized cysteine or CSSC) is added to fulfill the sulfur requirement ${ }^{32,36}$. In

97 keeping with this, a USA300 LAC strain of $S$. aureus (JE2) fails to proliferate in PN devoid of

98 CSSC (Fig. 1a). Notably, replacing CSSC with either $50 \mu \mathrm{M}$ GSH or $25 \mu \mathrm{M}$ GSSG stimulates $S$.

99 aureus proliferation (Fig. 1a). S. aureus utilization of GSSG expands the number of sulfur- 
containing metabolites present in host tissues that support proliferation of this pathogen. To

101 determine whether utilization of GSSG is conserved throughout the species, we examined

102 proliferation of clinical isolates in GSSG-supplemented medium. Growth of methicillin-sensitive

103 (MSSA) and methicillin-resistant (MRSA) clinical isolates was quantified in PN supplemented with

104 GSSG as the sole sulfur source. Compared to PN lacking a viable sulfur source, GSSG

105 supplementation substantially increased terminal $\mathrm{OD}_{600}$ (Fig. 1b and Supplementary Fig. S1).

106 Additionally, GSSG supplementation also considerably increased the terminal $\mathrm{OD}_{600}$ of other $S$.

107 aureus strains (Fig. 1c and Supplementary Fig. S1).

The SAUSA300_0200-0204 locus supports S. aureus utilization of GSH and GSSG as sulfur

110 sources. To determine genetic factors required for S. aureus utilization of GSSG as a sulfur

111 source, we screened the Nebraska Transposon Mutant Library for mutants that fail to proliferate

112 in PN medium supplemented with $25 \mu \mathrm{M}$ GSSG as the sole source of sulfur ${ }^{37}$. Five GSSG

113 proliferation-impaired mutants were identified in the screen, each harboring an independent

114 transposon insertion in one of five genes present in the SAUSA300_0200-ggt locus (Fig. 2a).

115 Notably, SAUSA300_0200-0203 encodes a putative nickel-peptide ABC transporter.

116 Backcrossing transposon-inactivated genes into an otherwise wild type (WT), isogenic JE2 strain

117 significantly decreased proliferation in medium supplemented with $25 \mu \mathrm{M}$ GSSG (Fig. 2b). The

118 transposon mutants also displayed decreased proliferation in PN supplemented with $50 \mu \mathrm{M}$ GSH

119 as the sole sulfur source (Fig. 2b). However, the backcrossed mutant strains demonstrated WT-

120 like growth in medium supplemented with $25 \mu \mathrm{M}$ CSSC or in a rich medium, indicating the

121 proliferation defect is specific to GSH and GSSG (Fig. 2b). To address complications associated

122 with auto-oxidation of GSH to GSSG in aerobic environments, we tested anaerobic proliferation

123 in media supplemented with GSH or GSSG. Mutant strains harboring a transposon in

124 SAUSA300_0201 or an in-frame deletion of all five genes were used in this assessment. In these

125 conditions, both mutant strains proliferate to WT levels in PN supplemented with CSSC but 
126 display reduced growth upon GSH or GSSG supplementation (Supplementary Fig. S2). This

127 finding confirms that SAUSA300_0200-ggt supports S. aureus utilization of both GSH and GSSG

128 as distinct sources of nutrient sulfur. Complementation experiments tested whether proliferation

129 of a ggt mutant cultured in PN medium supplemented with GSH or GSSG could be restored by

130 providing WT or a C-terminal His-tagged ggt encoded on a plasmid. ggt mutant strains harboring

131 either plasmid exhibit WT-like growth, confirming that failure to proliferate in GSSG- or GSH-

132 supplemented medium is due to genetic inactivation of ggt (Supplementary Fig. S3).

133 Based on the inability of the mutant strains to proliferate in GSH- or GSSG-supplemented

134 PN medium and the fact that SAUSA300_0200-SAUSA300_0203 encodes a putative ABC-

135 transporter with a predicted Ggt (SAUSA300_0204), we named these genes the glutathione

136 import system (gisABCD-ggt) (Fig. 2a). Domain architecture analysis of the GisABCD-Ggt system

137 reveals that GisA contains ATP-binding cassette domains (Supplementary Fig. S4). In support

138 of the annotation, recombinant GisA purified from Escherichia coli demonstrates ATP hydrolysis

139 activity (Supplementary Fig. S5). GisB and GisC are transmembrane permeases with nine

140 predicted transmembrane segments while GisD contains a signal peptide with a putative lipid

141 attachment site (Supplementary Fig. S4). Pfam analysis predicts that Ggt is a Y-glutamyl

142 transpeptidase (Supplementary Fig. S4).

144 Ggt hydrolyzes GSH and GSSG y-bonds and is cell associated. A hallmark of $\mathrm{y}$-glutamyl

145 transpeptidase is its capacity to cleave the GSH $\mathrm{y}$-bond, liberating glutamate. To validate the

146 Pfam domain prediction, we sought to determine whether $S$. aureus Ggt cleaves the $\mathrm{Y}$-bond

147 linking glutamate to Cys in GSH and GSSG ${ }^{38,39}$. C-terminal His-tagged recombinant Ggt (rGgt)

148 was expressed and purified from E. coli (Supplementary Fig. S6). In other species, Ggt enzymes

149 are translated as an inactive polypeptide that is auto-catalytically cleaved to generate approximate

$15040 \mathrm{kDa}$ and $35 \mathrm{kDa}$ subunits ${ }^{39,40}$. Consistent with this, a tripartite banding pattern consisting of

151 full-length pro-Ggt $(75 \mathrm{kDa})$ and smaller, mature enzyme subunits (40 kDa and $35 \mathrm{kDa})$ are 
152 observed (Supplementary Fig. S6). Mature Ggt cleaves GSH by attacking the glutamyl residue,

153 transferring it to the enzyme. Ultimately, water hydrolyzes the $\mathrm{y}$-bond, liberating glutamate ${ }^{41}$.

154 Therefore, to quantify S. aureus Ggt y-glutamyl transpeptidase activity, rGgt was incubated with

155 increasing concentrations of GSH or GSSG and glutamate release was measured by mass

156 spectrometry. Glutamate was detected in reactions containing rGgt incubated in the presence of

157 either GSH or GSSG (Fig. 3a). Importantly, glutamate was not detected in reactions lacking

158 substrate or rGgt, indicating glutamate release resulted from enzymatic activity (data not shown).

$159 \mathrm{~K}_{\mathrm{m}}$ values of Ggt for GSSG and GSH were determined to be $39 \mu \mathrm{M}$ and $58.5 \mu \mathrm{M}$, respectively.

160 These values are similar to previously reported Ggt homologues expressed in other organisms.

161 For example, the E. coli Ggt GSH $\mathrm{K}_{\mathrm{m}}$ value is $29 \mu \mathrm{M}^{42}$. These data support the in silico prediction

162 and provide a molecular explanation for the ggt mutant proliferation defect in medium

163 supplemented with GSH or GSSG as sources of nutrient sulfur-ggt mutant cells are unable to

164 initiate liberation of Cys due to an inability to hydrolyze the GSH or GSSG y-bond. Next, we

165 attempted to determine whether hydrolysis of GSH and GSSG occurs intracellularly or

extracellularly by localizing Ggt to $S$. aureus subcellular fractions.

Bacillus sp. secrete Ggt; however, structural predictions of $S$. aureus Ggt failed to detect canonical secretion signal sequences within the primary sequence (Supplementary Fig. S4). For example, SignalP-5.0 predictions detected considerably low likelihoods of signal peptide, twin-

170 arginine translocation (TAT) signal peptide, or lipoprotein signal peptide sequences $(0.007,0.003$,

1710.008 , respectively). TatP 1.0 also failed to predict a signal peptide ${ }^{43,44}$. Ggt localization varies

172 depending on the organism, but here it has implications for the substrate of GisABCD.

173 Extracellular Ggt supports a model whereby GisABCD imports Ggt cleavage products, whereas

174 intracellular Ggt suggests GisABCD imports GSH and GSSG intact ${ }^{18,19}$. We used the previously

175 described His-tagged Ggt expression vector (Ggt-His) that functionally complements the ggt::Tn

176 mutant (Supplementary Fig. S3) to determine subcellular localization of the enzyme. S. aureus

177 cells expressing native or Ggt-His were cultured in PN supplemented with $25 \mu \mathrm{M}$ GSSG, 
178 collected, and fractionated into supernatant and whole cell lysate (WCL) samples. An a-6xHis 179 antibody was used to monitor Ggt-His within each fraction. rGgt served as a size comparison 180 control. Ggt-His was not detected in the supernatant fractions; however, a band at $\sim 35 \mathrm{kDa}$ was 181 observed in both the Ggt-His WCL and rGgt samples. This band is specific to Ggt-His as it was 182 not observed in WCL generated from cells expressing Ggt lacking the His-tag and corresponds 183 to the rGgt subunit containing the His-tag. Presence of Ggt-His signal within the WCL fraction 184 supports the conclusion that Ggt is cell-associated (Fig. $\mathbf{3 b}$ ). WCL was further fractioned by 185 removal of the peptidoglycan (PG). Resulting protoplasts were lysed to generate a fraction 186 containing cytoplasm and membranes $(\mathrm{C}+\mathrm{M})$. His-dependent $\sim 35 \mathrm{kDa}$ signal was increasingly 187 apparent in the $\mathrm{C}+\mathrm{M}$ fraction compared to the $\mathrm{PG}$ fraction when equivalent levels of total protein 188 are assessed (Fig. 3b and 3c). Overall, the lack of a secretion signal sequence and enrichment 189 in the C+M fraction support a model whereby GSH and GSSG are imported intact and catabolized 190 in cytoplasm, leading to the eventual liberation of Cys to satisfy the nutrient sulfur requirement

191 (Supplementary Fig. S7). Cytoplasmic localization of Ggt has been reported in only one other 192 bacterial pathogen, Neisseria meningitidis ${ }^{28}$. Given that $S$. aureus does not synthesize GSH and 193 therefore does not utilize GSH as its major low molecular redox thiol, we predict that intracellular 194 Ggt will be associated with other bacterial species that lack GSH synthesis. Additionally, we 195 surmise that it will function primarily to satisfy the demand for nutrient sulfur in these species ${ }^{45,46}$.

197 GisABCD-Ggt is not required for systemic infection of S. aureus. We next tested whether

198 GisABCD-Ggt is important for $S$. aureus pathogenesis using a systemic murine model of infection.

199 However, organs of mice infected with a gisB::Tn mutant contained equivalent bacterial burdens 200 compared to WT-infected animals (Supplementary Fig. S8). This result indicates that GisABCD-

201 Ggt is dispensable for S. aureus pathogenesis during systemic infection. A possible explanation 202 for the lack of a virulence defect is that S. aureus may encode multiple GSH and GSSG 203 transporters. In fact, while GisABCD-Ggt supports proliferation of $S$. aureus in micromolar 
concentrations of GSSG or GSH, increasing GSH concentrations closer to those present in host tissues restores $\triangle$ gis $A B C D$-ggt mutant proliferation in PN medium (Supplementary Fig. S9). Increasing GSSG concentrations did not stimulate $\triangle$ gis $A B C D$-ggt mutant proliferation. These results support the conclusion that GisABCD-Ggt is an absolute requirement for GSSG utilization but that another, potentially low affinity, GSH transporter is also active in this pathogen.

211 for GisABCD-Ggt beyond systemic host colonization, we quantified conservation of the system

212 throughout Firmicutes. Due to the ubiquity of ABC-transporters across bacterial genera, we first

213 focused on potential Ggt homologues. We found that numerous Firmicutes encode Ggt

214 homologues, including Bacillus, Gracilibacillus, Lysinibacillus, and Brevibacterium; however,

215 subsequent identification of potential GisABCD homologues was limited to a minority of these

216 bacteria (Supplementary Fig. S10). Overall distribution of GisABCD-Ggt homologues across

217 Firmicutes revealed six distinct clusters. Cluster 3 is the least populated, as species in this cluster

218 encode only Ggt homologues. Firmicutes in clusters 2 and 4 contain Ggt and either a GisB or a

219 GisC homologue, respectively. Cluster 5 contains bacteria that encode GisB, GisC, and Ggt

220 homologues (Supplementary Fig. S10). Cluster 6 includes a few bacilli species that encode

221 proteins similar to the complete $S$. aureus GisABCD-Ggt system, but Cluster 1 stands apart as it

222 encompasses the $S$. aureus query sequences and comprises species that harbor complete

223 GisABCD-Ggt systems at greater than $>80 \%$ similarity. Notably, this cluster is distinct from other

224 Firmicutes and is restricted to members of the Staphylococcus genus with putative operons

225 encoding proteins most similar to S. aureus GisABCD-Ggt (Supplementary Fig. S10).

We predicted that GisABCD-Ggt would be widespread throughout the genus

227 Staphylococcus. Yet, only species in the S. aureus-related cluster complex-which includes

228 Staphylococcus argenteus, Staphylococcus schweitzeri, and Staphylococcus simiae-encode

229 homologues of a complete GisABCD-Ggt system (Fig. 4a) ${ }^{47,48}$. Conservation rapidly diverged as 
230 the next related species, Staphylococcus epidermidis, encodes apparent GisA, GisC, and Ggt

231 homologues with exceedingly low percent similarities (Fig. 4a) ${ }^{48}$. Lack of GisABCD-Ggt

232 conservation supports the hypothesis that $S$. epidermidis is incapable of utilizing low 233 concentrations of GSH as a source of nutrient sulfur.

GisABCD-Ggt promotes interspecies Staphylococcus competition in a GSH-specific

manner. To quantify S. epidermidis sulfur source dependent proliferation, we first needed to

237 define whether the organism is capable of utilizing Met, a component of PN medium, as a source

238 of nutrient sulfur. Additionally, S. epidermidis encodes predicted sulfate assimilation enzymes;

239 thus, sulfate $\left(\mathrm{MgSO}_{4}\right)$ was removed from $\mathrm{PN}$ medium ${ }^{49,50}$. Supplementation of sulfate-depleted

240 PN with Met as the sole source of nutrient sulfur stimulates proliferation of S. epidermidis, but not

241 S. aureus (Supplementary Fig. S11a). Therefore, Met was also removed and the resulting

242 medium, $\mathrm{PN}_{\text {mod, }}$, was used to quantify GSH- and GSSG-dependent proliferation of S. epidermidis

243 compared to $S$. aureus. Consistent with the importance of GisABCD-Ggt to S. aureus GSSG

244 utilization, supplementation of $25 \mu \mathrm{M}$ GSSG failed to stimulate appreciable proliferation of $S$.

245 epidermidis. Supplementation with $50 \mu \mathrm{M}$ GSH promoted delayed growth of S. epidermidis

246 relative to $S$. aureus (Supplementary Fig. S11a). Increasing GSH concentrations to $750 \mu \mathrm{M}$

247 resulted in comparable proliferation between S. epidermidis and S. aureus (Supplementary Fig.

248 S11a). These data indicate potential conservation of the unknown GSH acquisition system

249 between the two species. S. epidermidis does not exhibit enhanced growth in $375 \mu \mathrm{M}$ GSSG

250 relative to $25 \mu \mathrm{M}$ GSSG (Supplementary Fig. S11a), a result similar to the $S$. aureus $\triangle$ gis $A B C D$ -

251 ggt mutant and consistent with the conclusion that GisABCD-Ggt is an absolute requirement for

252 GSSG utilization in Staphylococcus.

253 Next, the capacity of GisABCD-Ggt to provide a competitive advantage to $S$. aureus over

254 S. epidermidis was determined. To test this, output CFU ratios of S. aureus to S. epidermidis after

255 a $24 \mathrm{~h}$ co-culture in $\mathrm{PN}_{\text {mod }}$ supplemented with different sulfur sources were quantified. S. aureus 
outcompeted both a $S$. epidermidis clinical isolate and the laboratory RP62a strain in $\mathrm{PN}_{\bmod }$

257 supplemented with $25 \mu \mathrm{M}$ GSSG or $50 \mu \mathrm{M}$ GSH (Fig. 4b, Supplementary Fig. S11b).

258 Conversely, S. epidermidis strains outcompeted S. aureus in medium with $50 \mu \mathrm{M}$ Met (Fig. 4b,

259 Supplementary Fig. S11b). S. aureus exhibited a competitive advantage over S. epidermidis in

$260750 \mu \mathrm{M} \mathrm{GSH}$, despite equivalent S. epidermidis proliferation in monoculture at this concentration

261 (Fig. 4b, Supplementary Fig. S11b). Both S. epidermidis strains outcompeted S. aureus $262 \Delta$ gisABCD-ggt in $25 \mu \mathrm{M}$ GSSG and $50 \mu \mathrm{M}$ GSH, underscoring the importance of GisABCD-Ggt

263 in promoting $S$. aureus competition over $S$. epidermidis in environments containing limiting

264 concentrations of GSH or GSSG. Equivalent quantities of $S$. epidermidis and S. aureus $265 \Delta$ gis $A B C D$-ggt were recovered in medium supplemented with $750 \mu \mathrm{M} \mathrm{GSH}$ (Fig. 4b,

266 Supplementary Fig. S11b). These findings provide additional support to the conclusion that Gis-

267 independent GSH acquisition is conserved between S. aureus and S. epidermidis.

Discussion

This study expands the number of host-derived metabolites that satisfy the $S$. aureus

271 nutrient sulfur requirement to include GSSG. Our results demonstrate that the ABC-transporter

272 system, GisABCD, and y-glutamyl transpeptidase, Ggt, support acquisition of GSH and GSSG as

273 sources of nutrient sulfur. We provide evidence that Ggt is cell associated. Cytoplasmic

274 localization of Ggt would be notable given that S. aureus does not utilize GSH as a low molecular

275 weight thiol and therefore suggests that the sole function of Ggt is nutrient sulfur catabolism.

276 However, our localization studies do not rule out the possibility that Ggt is anchored to the outer

277 leaflet of the plasma membrane. This localization pattern would support a model whereby GSH

278 or GSSG cleavage occurs first and is followed by transport of the Cys-Gly product through

279 GisABCD. Additional studies are required to corroborate the lack of canonical secretion

280 sequences and confirm intracellular Ggt localization. Nonetheless, we show that GisABCD-Ggt is

281 tuned to capture and catabolize exogenous GSH and GSSG for use as sources of nutrient sulfur. 
Failure of the gisB::Tn mutant to exhibit an overt virulence defect is partially explained by

283 the finding that in vitro supplementation with GSH concentrations mimicking those present in host

284 tissues stimulates gis mutant proliferation. This result is consistent with the fact that the host

285 invests considerable energy preserving redox homeostasis by maintaining GSH quantities in vast

286 excess to GSSG $^{9,51}$. Therefore, we demonstrate that while $S$. aureus utilizes the specialized

287 GisABCD-Ggt system to scavenge GSH and limiting GSSG, the pathogen has also evolved 288 multiple strategies to target the more abundant GSH.

289 Conservation of GisABCD-Ggt revealed the system is encoded in an exclusive clade of

290 Staphylococcus species that includes S. argenteus, S. schweitzeri, and S. simiae, but not S.

291 epidermidis. The fact that S. epidermidis proliferates poorly in a GSSG-supplemented medium

292 and that GisABCD-Ggt promotes S. aureus competition over S. epidermidis in GSH- and GSSG-

293 limiting environments further underscores the importance of GisABCD-Ggt to acquisition of GSSG

294 and GSH as sources of nutrient sulfur. S. argenteus colonizes fruit bats and monkeys and causes

295 skin and soft tissue infections and sepsis in humans ${ }^{52-59}$. S. epidermidis, S. aureus, and $S$.

296 schweitzeri colonize nasal passages of humans or closely related primates, but the lack of

297 GisABCD-Ggt conservation in S. epidermidis implies the system is not essential for nasal

298 colonization ${ }^{47,60-62}$. However, both S. aureus and S. epidermidis are also common residents of the

299 human skin microflora. Therefore, this environment could be further explored to determine

300 whether GisABCD-Ggt provides a competitive advantage to $S$. aureus and S. argenteus over $S$.

301 epidermidis in this dynamic host niche. 


\section{Materials and Methods}

303 Bacterial strains used in this study. The WT S. aureus strain used in these studies was JE2, a

304 laboratory derivative from the community-acquired, methicillin-resistant USA300 LAC ${ }^{63}$. 305 SAUSA300_0200-ggt mutant strains were generated via transduction of the transposon306 inactivated gene from the Nebraska Transposon Mutant Library strain into JE2 using previously 307 described techniques ${ }^{63,64}$. Bacterial strains used in this study are presented in Supplementary 308 Table S1.

A strain harboring an in-frame deletion of gis $A B C D$-ggt was constructed using a previously

310 described allelic exchange methodology for S. aureus ${ }^{65}$. One kb upstream of SAUSA300_0200

311 (gisA) and one kb downstream of ggt were amplified using primers listed in Supplementary Table

312 S2 and cloned into pKOR1-mcs. pKOR1-mcs was confirmed to have correct $1 \mathrm{~kb}$ homology

313 sequences by Sanger sequencing. The deletion strain was screened for hemolysis on blood agar

314 plates and displayed WT-like hemolysis.

Sulfur source dependent proliferation analysis. Chemically defined (PN) medium was

317 prepared as previously described ${ }^{35,66}$. PN medium was supplemented with $5 \mathrm{mg} \mathrm{mL}^{-1}$ glucose for

318 this work. Prior to inoculation in PN S. aureus was cultured in tryptic soy broth (TSB) overnight,

319 washed with PBS, and resuspended in PN medium to an $\mathrm{OD}_{600}$ equal to 1. Round-bottom 96-well

320 plates containing PN with $5 \mathrm{mg} \mathrm{mL}^{-1}$ glucose supplemented with the indicated sulfur sources were

321 inoculated with $S$. aureus strains at an initial inoculum of $\mathrm{OD}_{600}$ of 0.01 . Growth analysis was

322 carried out in a Biotek Epoch 2 plate reader set to $37^{\circ} \mathrm{C}$ with continuous shaking for the indicated

323 time. PN was modified to test sulfur source dependent proliferation of $S$. epidermidis and $S$.

324 aureus by replacing $\mathrm{MgSO}_{4}$ with $\mathrm{MgCl}_{2}$ and omitting Met, resulting in $\mathrm{PN}_{\text {mod. }}$ S. aureus and $S$.

325 epidermidis growth curves were performed as described above in $\mathrm{PN}_{\text {mod }}$ supplemented with the

326 indicated sulfur sources for $25 \mathrm{~h}$. Sulfur sources were purchased from Millipore Sigma and GSH

327 solutions were freshly prepared prior to each trial to limit oxidation. Alternatively, to ensure CSSC, 
$328 \mathrm{GSH}$, and GSSG were maintained in their respective reduced or oxidized forms, stock solutions

329 were prepared by weighing the appropriate amount of the chemical aerobically and immediately

330 transferring it to an anaerobic chamber (Coy) with a 95\%:5\% nitrogen:hydrogen atmosphere.

331 Sulfur sources were then resuspended in either anaerobically acclimated water (GSH and GSSG)

332 or anaerobically acclimated $1 \mathrm{~N} \mathrm{HCl}$ (CSSC). Anaerobic proliferation was monitored with the Coy

333 chamber using a Biotek Epoch 2 plate reader.

335 Isolation and growth phenotypes of clinical isolates. Four clinical isolates of $S$. aureus were 336 obtained from de-identified specimens at a regional hospital clinical laboratory. Three abscess

337 isolates were confirmed to be methicillin-resistant (strains 1055-1057) and the other was a 338 methicillin-sensitive bone isolate (strain 1059). Identification and minimum inhibitory 339 concentration assays were performed following Clinical and Laboratory Standards Institute 340 approved methods. After initial isolation, subcultures were grown on tryptic soy agar overnight.

Construction of pET28b::ggt and pET28b::gisA and purification of tagged protein. ggt and

343 gis $A$ open reading frames prior to the stop codon were amplified with primers sets listed in Table 344 S2. Expression vector pET28-b was digested with Ncol-HF and Xhol-HF. Plasmid assembly was 345 performed using Gibson assembly and the NEB HiFi assembly kit (NEB, New England, MA). The 346 assembly mixture was transformed into E. coli, cells were recovered in lysogeny broth (LB), and 347 plated onto LB agar containing $50 \mathrm{mg} \mathrm{mL}^{-1}$ kanamycin and $5 \mathrm{mg} \mathrm{mL}^{-1}$ glucose. Plasmids were 348 confirmed using Sanger sequencing and transformed into a NEB strain 3016 E. coli slyD mutant ${ }^{68}$.

349 Transformed E. coli were cultured in LB with $50 \mathrm{mg} \mathrm{mL}^{-1}$ kanamycin overnight at $37^{\circ} \mathrm{C}$ with 350 shaking at $225 \mathrm{rpm}$, sub-cultured 1:50 into $500 \mathrm{~mL} \mathrm{LB}$ with $50 \mathrm{mg} \mathrm{mL}^{-1}$ kanamycin in a $2 \mathrm{~L}$ flask 351 and grown to an $\mathrm{OD}_{600}$ of $0.4-0.7$. Ggt or GisA protein expression was induced by addition of 200 $352 \mu \mathrm{M}$ isopropyl-1-thio- $\beta$-D-galactopyranoside (IPTG) and the culture was separated into five 500 $353 \mathrm{~mL}$ flasks containing $100 \mathrm{~mL}$ of culture and incubated for $4 \mathrm{~h}$ at $27^{\circ} \mathrm{C}$ and $225 \mathrm{rpm}$ shaking. After 
354 induction, cells were centrifuged at $10,000 \times \mathrm{g}$ for $10 \mathrm{~min}$ at $4^{\circ} \mathrm{C}$ and washed with PBS. Resulting

355 GisA and Ggt induction pellets were resuspended in $40 \mathrm{~mL}$ of buffer containing $50 \mathrm{mM}$ tris, 200

$356 \mathrm{mM} \mathrm{KCl}, 20 \mathrm{mM}$ imidazole at $\mathrm{pH}$ 8, or $40-\mathrm{mL}$ buffer containing $50 \mathrm{mM}$ tris, $500 \mathrm{mM} \mathrm{NaCl}, 20 \mathrm{mM}$

357 imidazole at pH 8, respectively. Cells were lysed using a fluidizer set to 20,000 psi and samples

358 were run through 5 times. Lysates were then centrifuged at $15,000 \times \mathrm{g}$ for 15 min to remove intact

359 cells and the resulting supernatant was retained. To purify the target proteins, Ni-NTA

360 chromatography was used. Purification was performed by incubating the cleared lysate with $1 \mathrm{~mL}$

361 Ni-NTA resin (Qiagen, Hilden, Germany) on a rotating platform at $4^{\circ} \mathrm{C}$ for $2 \mathrm{~h}$. Protein was eluted

362 with $50 \mathrm{mM}$ tris $400 \mathrm{mM}$ imidazole. Buffers used to purify GisA contained $200 \mathrm{mM} \mathrm{KCl}$ while

363 buffers used to purify Ggt contained $500 \mathrm{mM} \mathrm{NaCl}$. Each buffer contained 1x protease inhibitor

364 cocktail (Millipore-Sigma). The GisA elutant was dialyzed using $10 \mathrm{mM}$ tris, $200 \mathrm{mM} \mathrm{KCl}$ at $\mathrm{pH}$

3657.5 as the dialysis buffer for $18 \mathrm{~h}$. The Ggt elutant was dialyzed using $10 \mathrm{mM}$ tris, $150 \mathrm{mM}$ at $\mathrm{pH}$

3667.0 as the dialysis buffer for $18 \mathrm{~h}$. Both the elutions were concentrated using $10 \mathrm{kDa}$ molecular

367 weight cutoff protein concentrators. Purification was confirmed via electrophoresis using $12 \%$

368 SDS-PAGE gels. Protein concentrations were determined with the bicinchoninic acid (BCA)

369 protein kit (BioRAD).

371 Quantitation of Ggt enzyme kinetics. Reactions were set up as follows: $10 \mathrm{mM}$ tris with 150

$372 \mathrm{mM} \mathrm{NaCl}$ containing $5 \mu \mathrm{g}$ recombinant Ggt and indicated concentrations of GSH and GSSG

373 dissolved in the reaction buffer. Reactions proceeded for $30 \mathrm{~min}$ at $37^{\circ} \mathrm{C}$ after which samples

374 were incubated at $80^{\circ} \mathrm{C}$ for $5 \mathrm{~min}$ to stop the reaction. Samples were dried using a roto-vac speed

375 vacuum and stored at $-80^{\circ} \mathrm{C}$ until they were hydrated via resuspension in water, derivatized with

376 carboxybenzyl (CBZ), and applied to a Waters Xevo TQ-S triple quadrupole mass spectrometer

377 as previously described ${ }^{69}$. Peak processing was performed by MAVEN, and the signal was 378 normalized to ${ }^{13} \mathrm{C}$-glutamine internal standard ${ }^{70}$. An external glutamate standard curve was 
379 generated using the same chromatographic conditions, and the signal was normalized to a ${ }^{13} \mathrm{C}$ -

380 glutamine internal standard. A fit equation to the standard curve was employed to quantitate 381 glutamate within the samples. Glutamate released per min was calculated and data were fit to the 382 Michaelis-Menten equation using GraphPad Prism. Data is the average of glutamate quantified 383 from four independent protein purifications.

Western blot analysis of S. aureus Ggt. The ggt ORF, his tag, and stop codon were amplified 386 from pET28b::ggt and cloned into pOS1 $\mathrm{P}_{\text {lgt }}$ digested with Ndel and HindIII using Gibson assembly 387 to generate Ggt-His. Additionally, the ggt ORF was amplified from JE2 and pOS1 $\mathrm{P}_{\text {lgt }}$ digested 388 with Ndel and HindlII using Gibson assembly to generate the untagged Ggt. Plasmids were 389 confirmed by Sanger sequencing and transformed from E. coli DH5a into S. aureus RN4220 via 390 electroporation. Plasmids were purified from RN4220 and transformed into JE2 ggt::Tn. An empty 391 vector control strain was generated by transforming JE2 and ggt::Tn with pOS1 $\mathrm{P}_{\text {lgt. }}$ To assess

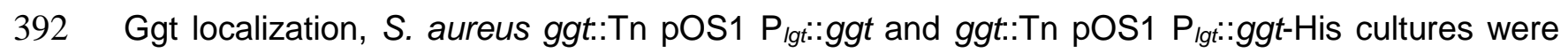
393 prepared as described in the proliferation analysis section and sub-cultured into 3, $250 \mathrm{~mL}$ flasks 394 each containing $100 \mathrm{~mL} P N$ with $25 \mu \mathrm{M}$ GSSG and $10 \mu \mathrm{g} \mathrm{mL}^{-1}$ chloramphenicol at a starting $\mathrm{OD}_{600}$ 395 equal to 0.1 . Cells were cultured $4 \mathrm{~h}$ at $37^{\circ} \mathrm{C}$ and $225 \mathrm{rpm}$ shaking. At this time cells reached 396 mid-exponential phase and were recovered via centrifugation, the supernatant retained, and the 397 pellet washed with PBS. Fifty $\mathrm{mL}$ of the initial supernatant was precipitated with trichloracetic acid 398 (final percent of $10 \% \mathrm{v} / \mathrm{v})(\mathrm{TCA})$, incubated $1 \mathrm{~h}$ to overnight at $4^{\circ} \mathrm{C}$, pelleted, and the resulting 399 pellet washed twice with $95 \%$ ethanol. Samples were mixed with Laemmli buffer, boiled for 10 400 min, run on a $12 \%$ SDS-PAGE gel using tris-glycine running buffer, and transferred at 65 volts for $4011 \mathrm{~h}$ at $4^{\circ} \mathrm{C}$ to PVDF membrane. Forty $\mu \mathrm{g}$ were loaded for WCL, PG, and $\mathrm{C}+\mathrm{M}$. Membranes were 402 incubated overnight in phosphate buffered saline tween-20 (PBST) with 3\% bovine serum albumin 403 (BSA) at $4^{\circ} \mathrm{C}$ with agitation. An $\alpha-H i s$ mouse antibody was used as the primary antibody at a 
1:4,000 dilution in PBST supplemented with 5\% BSA and incubated for $1 \mathrm{~h}$ with shaking. The membrane was washed thrice with PBST. An a-mouse IgG conjugated to horseradish peroxidase (HRP) was used as the secondary antibody at a dilution of 1:4,000 (Sigma-Aldrich). To assess cell wall fractionation a mouse $\alpha$-protein A (Spa) primary antibody was used at 1:6,000 dilution. Membranes were washed thrice in PBST after incubation with secondary antibody. Membranes were developed using the ECL Prime kit (Cytiva, Marlborough, MA) and imaged using Amersham

410 Imager 600 (GE Healthcare, Amersham, Buckinghamshire, UK). Fractionation was repeated with

4113 independent sets of cultures and one set of immunoblots is presented as a representative.

413 Identification of GisABCD-Ggt homologues across bacteria. The USA300_FPR3757

414 (assembly GCF_000013465.1) Ggt protein sequence (ABD22038.1) was used as the query

415 protein for homology searches (using DELTA-BLAST) using the NCBI RefSeq database ${ }^{69-71}$. Data

416 was filtered to include only Firmicutes that encoded Ggt homologues containing a glutamyl

417 transpeptidase domain. This dataset was used as the subject to query USA300_FPR3757

418 GisABCD using DELTA-BLAST. Percent similarities to the $S$. aureus GisABCD-Ggt protein amino 419 acid sequences were used to generate a heatmap. The heatmap and hierarchical clustering of 420 similar protein profiles were generated using the R package, pheatmap.

422 Quantitation of S. aureus and S. epidermidis competition. S. aureus, S. aureus $\triangle$ gis $A B C D-$ 423 ggt, and S. epidermidis were cultured overnight in TSB, pelleted, wash in PBS, and normalized 424 to the same $\mathrm{OD}_{600}$ in $P N_{\text {mod. }}$. Strains were mixed in a 1:1 ratio (v/v) and inoculated into $5 \mathrm{~mL} P N_{\text {mod. }}$ $425 \mathrm{PN}_{\text {mod }}$ was supplemented with $25 \mu \mathrm{M}$ GSSG, $50 \mu \mathrm{M}$ GSH, $750 \mu \mathrm{M}$ GSH, or $50 \mu \mathrm{M}$ Met. Dilution 426 plating of the freshly mixed co-culture was plated onto mannitol salt agar (MSA) to quantify initial 427 counts of each organism. Cultures were incubated for $24 \mathrm{~h}$ at $37^{\circ} \mathrm{C}$ with $225 \mathrm{rpm}$ shaking after 428 which the cultures were dilution plated onto MSA and allowed to grow for $48 \mathrm{~h}$ at $35^{\circ} \mathrm{C}$. S. aureus 
429 ferments mannitol and appears yellow on MSA, while S. epidermidis does not and maintains a

430 pink color; consequently, yellow and pink colored colonies were enumerated to assess quantities

431 of each organism. Competitive indices $(\mathrm{Cl})$ were calculated by dividing the $S$. aureus to $S$.

432 epidermidis output ratio by the $S$. aureus to $S$. epidermidis input ratio. A $\mathrm{Cl}$ greater than one

433 indicates more $S$. aureus than $S$. epidermidis while a $\mathrm{Cl}$ less than one signifies greater quantities

434 of $S$. epidermidis compared to $S$. aureus.

\section{Acknowledgements}

437 The defined transposon mutant library used in this study was provided by the Network on

438 Antimicrobial Resistance in Staphylococcus aureus (NARSA) for distribution by BEI Resources,

439 NIAID, NIH: Nebraska Transposon Mutant Library (NTML) Screening Array NR-48501. We thank

440 the laboratory of Dr. Taeok Bae at Indiana University for supplying the pKOR1-mcs plasmid, Dr.

441 Anthony Richardson for the S. epidermidis strain RP62a, and we thank the Dr. Victor DiRita and

442 Dr. Sean Crosson laboratories at Michigan State University for technical support. We thank Dr.

443 Martin P. Ogrodzinski and the MSU Mass Spectrometry and Metabolomics Core for technical

444 support. This work is funded by the National Institutes of Health R01 Al139074 and R21 Al142517. 


\section{References}

1. Skaar, E. P., Humayun, M., Bae, T., DeBord, K. L. \& Schneewind, O. Iron-source preference of Staphylococcus aureus infections. Science 305, 1626-1628 (2004).

2. Mazmanian, S. K. et al. Passage of heme-iron across the envelope of Staphylococcus aureus. Science 299, 906-909 (2003).

3. Lensmire, J. M. \& Hammer, N. D. Nutrient sulfur acquisition strategies employed by bacterial pathogens. Curr. Opin. Microbiol. 47, 52-58 (2018).

4. Beinert, H. A tribute to sulfur. Eur. J. Biochem. 267, 5657-5664 (2000).

5. Ayala-Castro, C., Saini, A. \& Outten, F. W. Fe-S cluster assembly pathways in bacteria. Microbiol. Mol. Biol. Rev. 72, 110-25, table of contents (2008).

6. Guédon, E. \& Martin-Verstraete, I. in Amino acid biosynthesis pathways, regulation and metabolic engineering (ed. Wendisch, V. F.) 195-218 (Springer Berlin Heidelberg, 2007). doi:10.1007/7171_2006_060

7. Kredich, N. M. in Escherichia coli and Salmonella, cellular and molecular biology (ed. Neidhardt) 514-527 (1996).

8. Lill, R. \& Mühlenhoff, U. Iron-sulfur protein biogenesis in eukaryotes: components and mechanisms. Annu. Rev. Cell Dev. Biol. 22, 457-486 (2006).

9. Meister, A. \& Anderson, M. E. Glutathione. Annu. Rev. Biochem. 52, 711-760 (1983).

10. Dubois, T. et al. Control of Clostridium difficile Physiopathology in Response to Cysteine Availability. Infect. Immun. 84, 2389-2405 (2016).

11. Stenson, T. H., Patton, A. K. \& Weiss, A. A. Reduced glutathione is required for pertussis toxin secretion by Bordetella pertussis. Infect. Immun. 71, 1316-1320 (2003).

12. Suzuki, H., Koyanagi, T., Izuka, S., Onishi, A. \& Kumagai, H. The yliA, -B, -C, and -D genes of Escherichia coli K-12 encode a novel glutathione importer with an ATP-binding cassette. J. Bacteriol. 187, 5861-5867 (2005).

13. Wang, Z., Zhang, M., Shi, X. \& Xiang, Q. Purification and Characterization of an ATPase GsiA from Salmonella enterica. Biomed Res. Int. 2017, 3076091 (2017).

14. Vergauwen, B., Elegheert, J., Dansercoer, A., Devreese, B. \& Savvides, S. N. Glutathione import in Haemophilus influenzae $\mathrm{Rd}$ is primed by the periplasmic heme-binding protein HbpA. Proc. Natl. Acad. Sci. USA 107, 13270-13275 (2010).

15. Vergauwen, B., Pauwels, F., Vaneechoutte, M. \& Van Beeumen, J. J. Exogenous glutathione completes the defense against oxidative stress in Haemophilus influenzae. J. Bacteriol. 185, 1572-1581 (2003).

16. Vergauwen, B. et al. Molecular and structural basis of glutathione import in Gram-positive bacteria via GshT and the cystine ABC importer TcyBC of Streptococcus mutans. Mol. Microbiol. 89, 288-303 (2013).

17. Potter, A. J., Trappetti, C. \& Paton, J. C. Streptococcus pneumoniae uses glutathione to defend against oxidative stress and metal ion toxicity. J. Bacteriol. 194, 6248-6254 (2012).

18. Alkhuder, K., Meibom, K. L., Dubail, I., Dupuis, M. \& Charbit, A. Glutathione provides a source of cysteine essential for intracellular multiplication of Francisella tularensis. PLoS Pathog. 5, e1000284 (2009).

19. Ramsey, K. M. et al. Tn-Seq reveals hidden complexity in the utilization of host-derived glutathione in Francisella tularensis. PLoS Pathog. 16, e1008566 (2020).

20. Masip, L., Veeravalli, K. \& Gerogiou, G. The Many Faces of Glutathione in Bacteria. Antioxid. Redox Signal. 8, 753-762 (2006).

21. Orlowski, M. \& Meister, A. The gamma-glutamyl cycle: a possible transport system for amino acids. Proc. Natl. Acad. Sci. USA 67, 1248-1255 (1970).

22. Tate, S. S. \& Meister, A. Y-Glutamyl transpeptidase: catalytic, structural and functional aspects. Mol. Cell. Biochem. 39, 357-368 (1981).

23. Cooper, A. J. Biochemistry of sulfur-containing amino acids. Annu. Rev. Biochem. 52, 187- 
222 (1983).

24. Suzuki, H., Kamatani, S., Kim, E. S. \& Kumagai, H. Aminopeptidases A, B, and N and dipeptidase $\mathrm{D}$ are the four cysteinylglycinases of Escherichia coli K-12. J. Bacteriol. 183, 1489-1490 (2001).

25. Frackenpohl, J., Arvidsson, P. I., Schreiber, J. V. \& Seebach, D. The Outstanding Biological Stability of $\beta$ - andy-Peptides toward Proteolytic Enzymes: An In Vitro Investigation with Fifteen Peptidases. Chembiochem 2, 445-455 (2001).

26. Suzuki, H., Kumagai, H. \& Tochikura, T. gamma-Glutamyltranspeptidase from Escherichia coli K-12: formation and localization. J. Bacteriol. 168, 1332-1335 (1986).

27. Xu, K. \& Strauch, M. A. Identification, sequence, and expression of the gene encoding gamma-glutamyltranspeptidase in Bacillus subtilis. J. Bacteriol. 178, 4319-4322 (1996).

28. Takahashi, H. \& Watanabe, H. Post-translational processing of Neisseria meningitidis gamma-glutamyl aminopeptidase and its association with inner membrane facing to the cytoplasmic space. FEMS Microbiol. Lett. 234, 27-35 (2004).

29. Kluytmans, J. A. et al. Nasal carriage of Staphylococcus aureus as a major risk factor for wound infections after cardiac surgery. J. Infect. Dis. 171, 216-219 (1995).

30. Gordon, R. J. \& Lowy, F. D. Pathogenesis of methicillin-resistant Staphylococcus aureus infection. Clin. Infect. Dis. 46 Suppl 5, S350-9 (2008).

31. Klevens, R. M. et al. Invasive methicillin-resistant Staphylococcus aureus infections in the United States. JAMA 298, 1763-1771 (2007).

32. Lithgow, J. K., Hayhurst, E. J., Cohen, G., Aharonowitz, Y. \& Foster, S. J. Role of a cysteine synthase in Staphylococcus aureus. J. Bacteriol. 186, 1579-1590 (2004).

33. Helmann, J. D. Bacillithiol, a new player in bacterial redox homeostasis. Antioxid. Redox Signal. 15, 123-133 (2011).

34. Wymann, M. P., von Tscharner, V., Deranleau, D. A. \& Baggiolini, M. The onset of the respiratory burst in human neutrophils. Real-time studies of $\mathrm{H} 2 \mathrm{O} 2$ formation reveal a rapid agonist-induced transduction process. J. Biol. Chem. 262, 12048-12053 (1987).

35. Pattee, P. A. \& Neveln, D. S. Transformation analysis of three linkage groups in Staphylococcus aureus. J. Bacteriol. 124, 201-211 (1975).

36. Soutourina, O. et al. CymR, the master regulator of cysteine metabolism in Staphylococcus aureus, controls host sulphur source utilization and plays a role in biofilm formation. Mol. Microbiol. 73, 194-211 (2009).

37. Bose, J. L., Fey, P. D. \& Bayles, K. W. Genetic tools to enhance the study of gene function and regulation in Staphylococcus aureus. Appl. Environ. Microbiol. 79, 2218-2224 (2013).

38. Oinonen, C. \& Rouvinen, J. Structural comparison of Ntn-hydrolases. Protein Sci. 9, 23292337 (2000).

39. Suzuki, H. \& Kumagai, H. Autocatalytic processing of gamma-glutamyltranspeptidase. J. Biol. Chem. 277, 43536-43543 (2002).

40. West, M. B. et al. Autocatalytic cleavage of human gamma-glutamyl transpeptidase is highly dependent on N-glycosylation at asparagine 95. J. Biol. Chem. 286, 28876-28888 (2011).

41. Okada, T., Suzuki, H., Wada, K., Kumagai, H. \& Fukuyama, K. Crystal structures of gamma-glutamyltranspeptidase from Escherichia coli, a key enzyme in glutathione metabolism, and its reaction intermediate. Proc. Natl. Acad. Sci. USA 103, 6471-6476 (2006).

42. Suzuki, H., Kumagai, H. \& Tochikura, T. gamma-Glutamyltranspeptidase from Escherichia coli K-12: purification and properties. J. Bacteriol. 168, 1325-1331 (1986).

43. Bendtsen, J. D., Nielsen, H., Widdick, D., Palmer, T. \& Brunak, S. Prediction of twinarginine signal peptides. BMC Bioinformatics 6, 167 (2005).

44. Armenteros, J. J. A. et al. SignalP 5.0 improves signal peptide predictions using deep neural networks. Nat. Biotechnol. 37, 420-423 (2019). 
45. Newton, G. L. et al. Bacillithiol is an antioxidant thiol produced in Bacilli. Nat. Chem. Biol. 5, 625-627 (2009).

46. Pöther, D.-C. et al. Distribution and infection-related functions of bacillithiol in Staphylococcus aureus. Int. J. Med. Microbiol. 303, 114-123 (2013).

47. Tong, S. Y. C. et al. Novel staphylococcal species that form part of a Staphylococcus aureus-related complex: the non-pigmented Staphylococcus argenteus sp. nov. and the non-human primate-associated Staphylococcus schweitzeri sp. nov. Int. J. Syst. Evol. Microbiol. 65, 15-22 (2015).

48. Thurlow, L. R., Stephens, A. C., Hurley, K. E. \& Richardson, A. R. Lack of nutritional immunity in diabetic skin infections promotes Staphylococcus aureus virulence. Sci. Adv. 6, (2020).

49. Kanehisa, M. \& Goto, S. KEGG: Kyoto encyclopedia of genes and genomes. Nucleic Acids Res. 28, 27-30 (2000).

50. Kanehisa, M., Furumichi, M., Sato, Y., Ishiguro-Watanabe, M. \& Tanabe, M. KEGG: integrating viruses and cellular organisms. Nucleic Acids Res. 49, D545-D551 (2021).

51. Franco, R., Schoneveld, O. J., Pappa, A. \& Panayiotidis, M. I. The central role of glutathione in the pathophysiology of human diseases. Arch Physiol Biochem 113, 234-258 (2007).

52. Schaumburg, F. et al. Staphylococcus aureus complex from animals and humans in three remote African regions. Clin. Microbiol. Infect. 21, 345.e1-8 (2015).

53. Okuda, K. V. et al. Molecular epidemiology of Staphylococcus aureus from Lambaréné, Gabon. Eur. J. Clin. Microbiol. Infect. Dis. 35, 1963-1973 (2016).

54. Ateba Ngoa, U. et al. Epidemiology and population structure of Staphylococcus aureus in various population groups from a rural and semi urban area in Gabon, Central Africa. Acta Trop. 124, 42-47 (2012).

55. Schaumburg, F. et al. Highly divergent Staphylococcus aureus isolates from African nonhuman primates. Environ. Microbiol. Rep. 4, 141-146 (2012).

56. Olatimehin, A. et al. Staphylococcus aureus Complex in the Straw-Colored Fruit Bat (Eidolon helvum) in Nigeria. Front. Microbiol. 9, 162 (2018).

57. Holt, D. C. et al. A very early-branching Staphylococcus aureus lineage lacking the carotenoid pigment staphyloxanthin. Genome Biol. Evol. 3, 881-895 (2011).

58. Ng, J. W. S. et al. Phylogenetically distinct Staphylococcus aureus lineage prevalent among indigenous communities in northern Australia. J. Clin. Microbiol. 47, 2295-2300 (2009).

59. Dupieux, C. et al. Community-acquired infections due to Staphylococcus argenteus lineage isolates harbouring the Panton-Valentine leucocidin, France, 2014. Euro Surveill. 20, (2015).

60. Gould, J. C. \& McKILLOP, E. J. The carriage of Staphylococcus pyogenes var. aureus in the human nose. J. Hyg. (Lond.) 52, 304-310 (1954).

61. Kluytmans, J., van Belkum, A. \& Verbrugh, H. Nasal carriage of Staphylococcus aureus: epidemiology, underlying mechanisms, and associated risks. Clin. Microbiol. Rev. 10, 505520 (1997).

62. Frank, D. N. et al. The human nasal microbiota and Staphylococcus aureus carriage. PLoS One 5, e10598 (2010).

63. Fey, P. D. et al. A genetic resource for rapid and comprehensive phenotype screening of nonessential Staphylococcus aureus genes. MBio 4, e00537-12 (2013).

64. Schneewind, O. \& Missiakas, D. Genetic manipulation of Staphylococcus aureus. Curr Protoc Microbiol 32, Unit 9C.3. (2014).

65. Bae, T. \& Schneewind, O. Allelic replacement in Staphylococcus aureus with inducible counter-selection. Plasmid 55, 58-63 (2006).

66. Lensmire, J. M. et al. The Staphylococcus aureus Cystine Transporters TcyABC and TcyP Facilitate Nutrient Sulfur Acquisition during Infection. Infect. Immun. 88, (2020). 
67. Kanehisa, M. Toward understanding the origin and evolution of cellular organisms. Protein Sci. 28, 1947-1951 (2019).

601

602

603

604

605

606

607

608

609

610

68. Hammer, N. D. et al. The C-terminal repeating units of CsgB direct bacterial functional amyloid nucleation. J. Mol. Biol. 422, 376-389 (2012).

69. Ogrodzinski, M. P. et al. Measuring the nutrient metabolism of adherent cells in culture. Methods Mol. Biol. 1862, 37-52 (2019).

70. Melamud, E., Vastag, L. \& Rabinowitz, J. D. Metabolomic analysis and visualization engine for LC-MS data. Anal. Chem. 82, 9818-9826 (2010).

71. Altschul, S. F., Gish, W., Miller, W., Myers, E. W. \& Lipman, D. J. Basic local alignment search tool. J. Mol. Biol. 215, 403-410 (1990).

72. Boratyn, G. M. et al. Domain enhanced lookup time accelerated BLAST. Biol. Direct 7, 12 (2012).

73. O'Leary, N. A. et al. Reference sequence (RefSeq) database at NCBI: current status, taxonomic expansion, and functional annotation. Nucleic Acids Res. 44, D733-45 (2016).

613

614

615 
616

617

618

619

620

\section{1}

\section{2}

623

624

625

\section{Figure Legends}

Figure 1. Supplementation of GSSG as the sole source of nutrient sulfur supports proliferation of S. aureus. a, WT JE2 S. aureus was cultured in PN medium lacking a viable sulfur source. CSSC, GSSG, or GSH were added to the medium at indicated concentrations. b, JE2 and either MSSA or MRSA clinical isolates were grown in PN supplemented without a viable sulfur source (None) or $25 \mu \mathrm{M}$ GSSG for 19 hours (h). c, S. aureus strains were cultured in PN medium supplemented without sulfur (None) or $25 \mu \mathrm{M}$ GSSG and cultured for $25 \mathrm{~h}$. Bars depict the mean terminal optical density at $600 \mathrm{~nm}\left(\mathrm{OD}_{600}\right)$, and circles represent individual replicate terminal $\mathrm{OD}_{600}$. The mean of at least three independent trials and error bars representing \pm 1 standard error of the mean are presented in $\mathbf{a}, \mathbf{b}$, and $\mathbf{c}$.

Figure 2. SAUSA300_0200-ggt supports S. aureus proliferation on GSH and GSSG as sources of nutrient sulfur. a, Orientation of SAUSA300_0200-ggt within the S. aureus genome. b, Strains cultured in tryptic soy broth (TSB) or PN supplemented with $25 \mu \mathrm{M}$ GSSG, $50 \mu \mathrm{M}$ GSH, or $25 \mu \mathrm{M}$ CSSC. The mean of at least three independent trials and error bars representing \pm 1 standard error of the mean are presented.

Figure 3. S. aureus Ggt liberates glutamate from GSH and GSSG and is cell-associated. a, rGgt was incubated in the presence of indicated GSH and GSSG concentrations. Mean glutamate release per minute was measured using four independent rGgt protein preparations. Glutamate released per min was calculated and the data was fit with the Michaelis Menten equation using GraphPad Prism. Error bars represent \pm standard error of the mean. $\mathbf{b}$ and $\mathbf{c}$, Fractions of $S$.

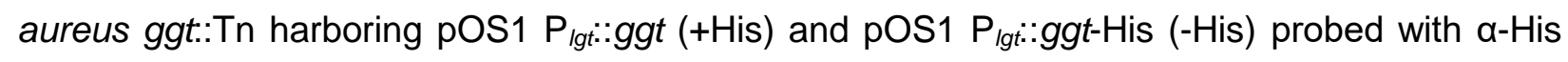
antibody. Fractions include the culture supernatant (sup), whole cell lysate (WCL), peptidoglycan $(P G)$, and protoplast lysate which contains the cytoplasm and membranes $(C+M)$. c, Fractions were probed with antibody against protein A ( $\alpha-S p a)$. 
644 Figure 4. GisABCD is conserved in exclusive staphylococci and promotes competition in

645 GSH- or GSSG-supplemented media. a, Heatmap depicting percent similarities of GisABCD-

646 Ggt proteins across Staphylococci (S. aureus Gis-Ggt proteins were used as the starting points

647 for the homology searches). b, in vitro competition experiments between $S$. aureus and $S$.

648 epidermidis strain RP62a. The line represents the mean competitive index for each individual trial.

649 Error bars represent \pm 1 standard error of the mean. * Indicates $p$-value $<0.05$ as determined by

650 one-way ANOVA with Tukey's multiple test correction. 
$\mathbf{a}$
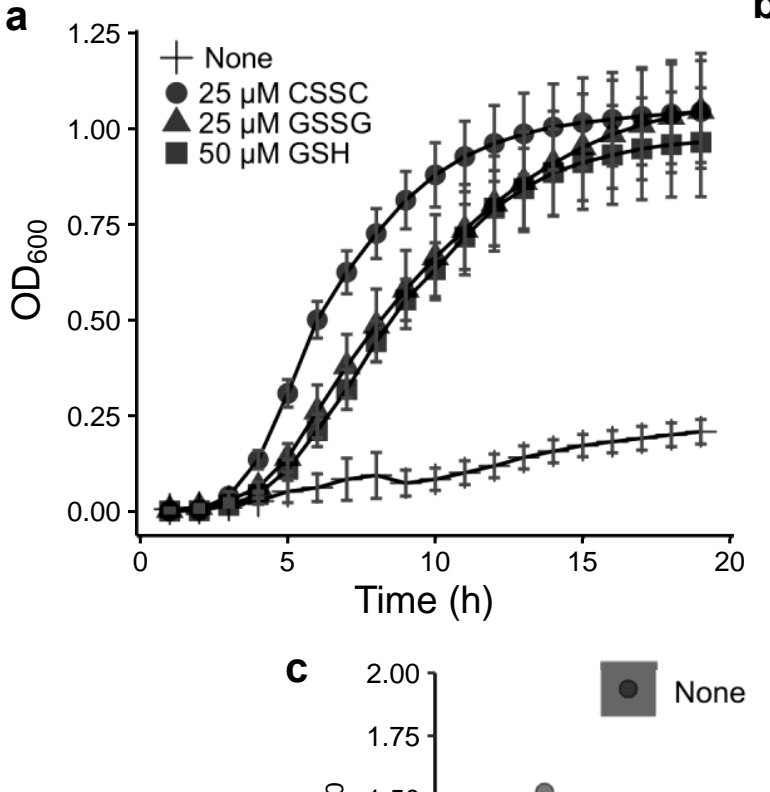

b

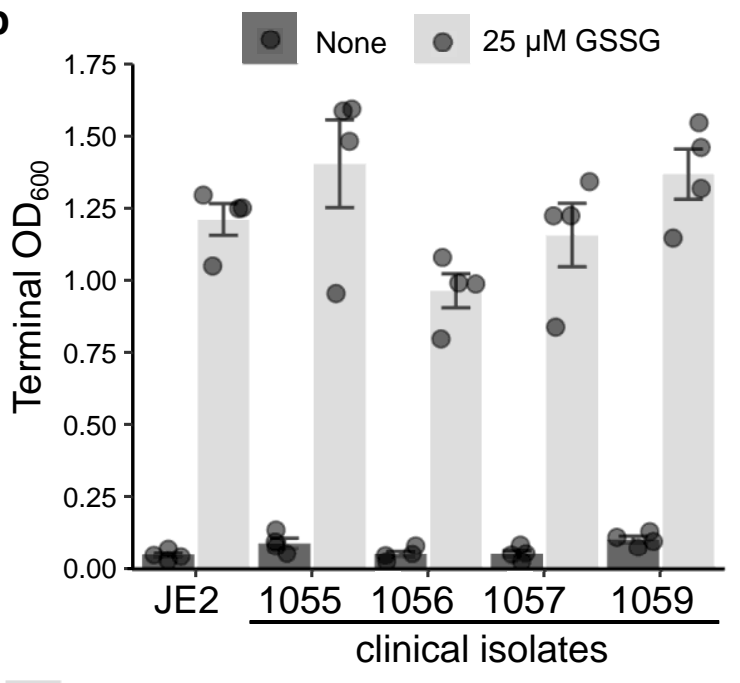

- $25 \mu \mathrm{M}$ GSSG

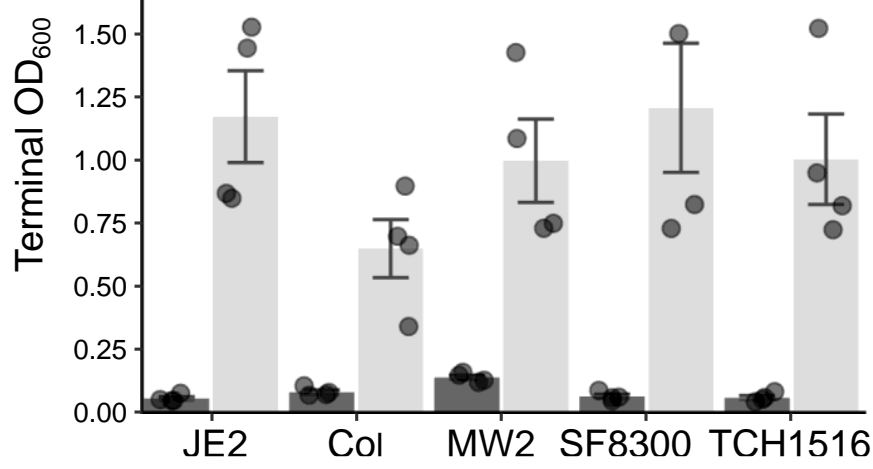

Figure 1. Supplementation of GSSG as the sole source of nutrient sulfur supports proliferation of $\boldsymbol{S}$. aureus. a, WT JE2 S. aureus was cultured in PN medium lacking a viable sulfur source. CSSC, GSSG, or GSH were added to the medium at indicated concentrations. b, JE2 and either MSSA or MRSA clinical isolates were grown in PN supplemented without a viable sulfur source (None) or $25 \mu \mathrm{M}$ GSSG for 19 hours (h). c, S. aureus strains were cultured in PN medium supplemented without sulfur (None) or $25 \mu \mathrm{M} \mathrm{GSSG}$ and cultured for $25 \mathrm{~h}$. Bars depict the mean terminal optical density at $600 \mathrm{~nm}\left(\mathrm{OD}_{600}\right)$, and circles represent individual replicate terminal $\mathrm{OD}_{600}$. The mean of at least three independent trials and error bars representing \pm 1 standard error of the mean are presented in $\mathbf{a}$, $\mathbf{b}$, and $\mathbf{c}$. 
a

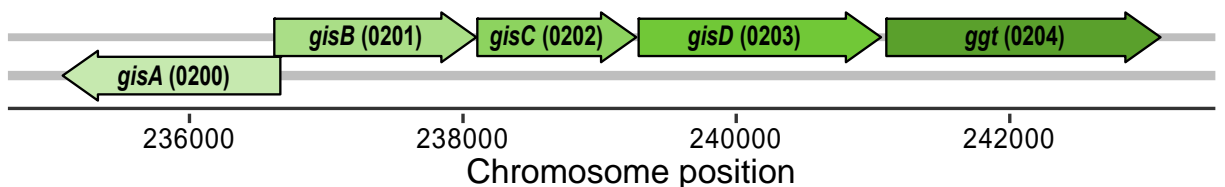

b
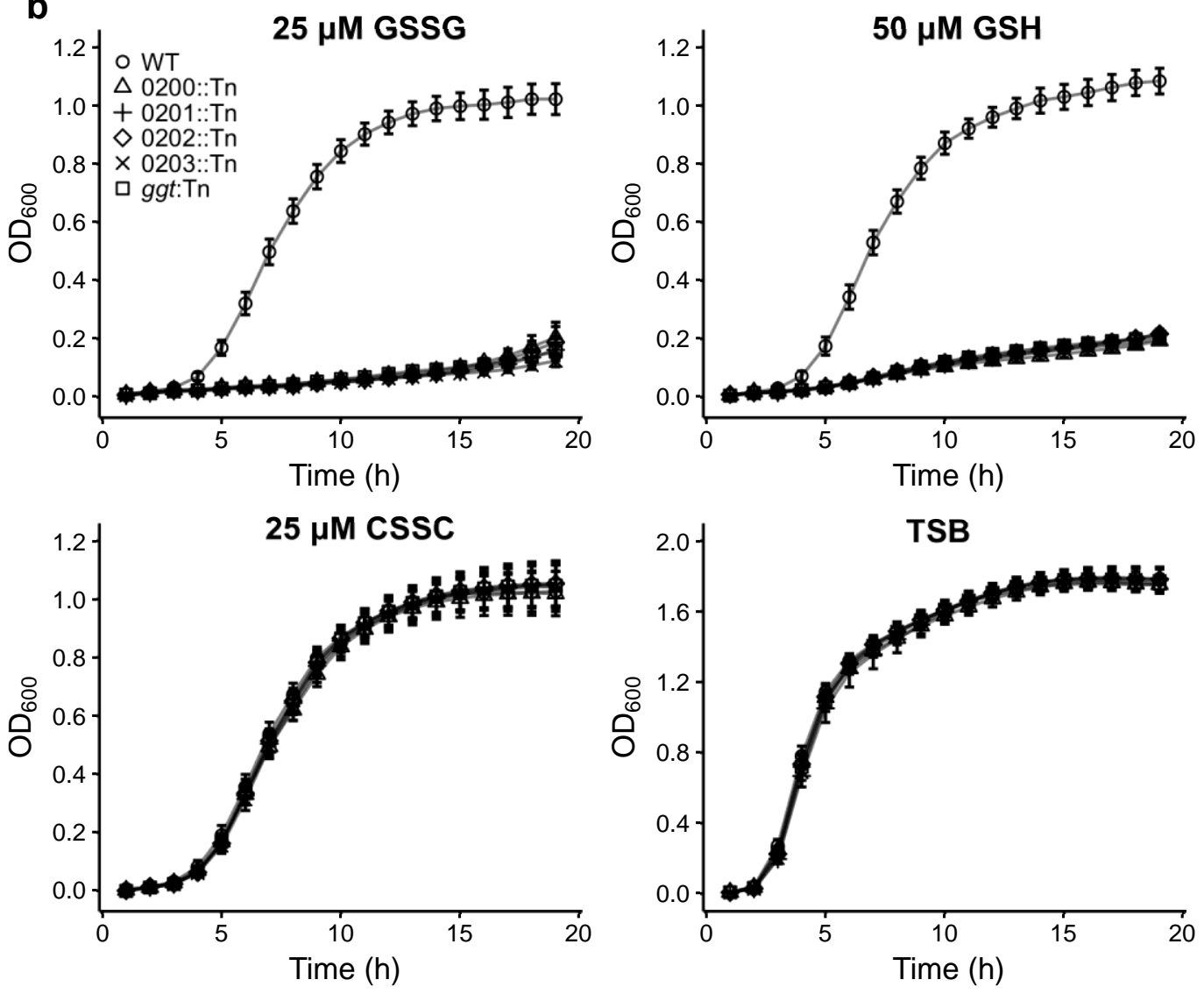

655

656

657

658

659

660

661
Figure 2. SAUSA300_0200-ggt supports S. aureus proliferation on GSH and GSSG as sources of nutrient sulfur. a, Orientation of SAUSA300_0200-ggt within the S. aureus genome. b, Strains cultured in tryptic soy broth (TSB) or PN supplemented with $25 \mu \mathrm{M}$ GSSG, $50 \mu \mathrm{M} \mathrm{GSH}$, or $25 \mu \mathrm{M}$ CSSC. The mean of at least three independent trials and error bars representing \pm 1 standard error of the mean are presented. 

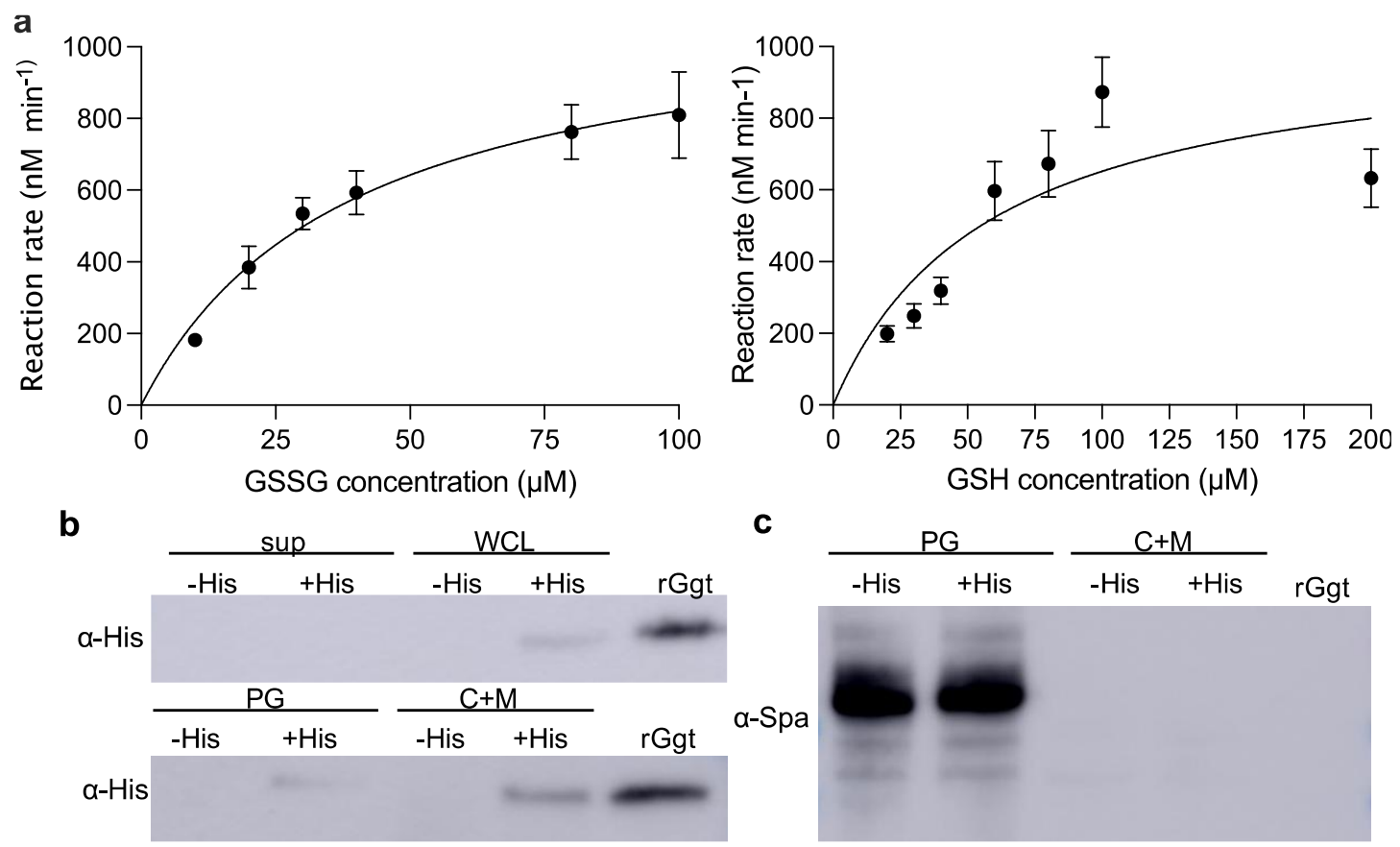

Figure 3. S. aureus Ggt liberates glutamate from GSH and GSSG and is cell-associated. a, rGgt was incubated in the presence of indicated GSH and GSSG concentrations. Mean glutamate release per minute was measured using four independent rGgt protein preparations. Glutamate released per min was calculated and the data was fit with the Michaelis Menten equation using GraphPad Prism. Error bars represent \pm standard error of the mean. $\mathbf{b}$ and $\mathbf{c}$, Fractions of $S$. aureus ggt::Tn harboring pOS1 $\mathrm{P}_{\text {lgt }}:$ ggt (-His) and pOS1 $\mathrm{P}_{\text {lgt }}:: g g t-\mathrm{His}(+\mathrm{His})$ probed with $\alpha$-His antibodies. Fractions include the culture supernatant (sup), whole cell lysate (WCL), peptidoglycan (PG), and protoplast lysate which contains the cytoplasm and membranes $(\mathrm{C}+\mathrm{M})$. c, Fractions were probed with anti-protein $\mathrm{A}(\mathrm{Spa})$ antibodies $(\alpha-\mathrm{Spa})$. 
a

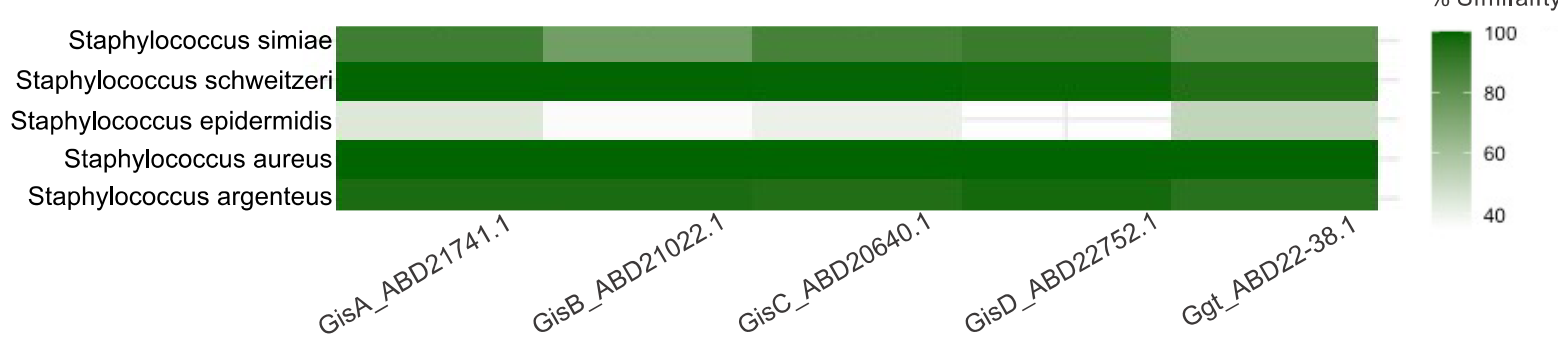

b

- S. aureus vs. S. epidermidis

- $\quad \triangle$ gisABCD-ggt vs. S. epidermidis

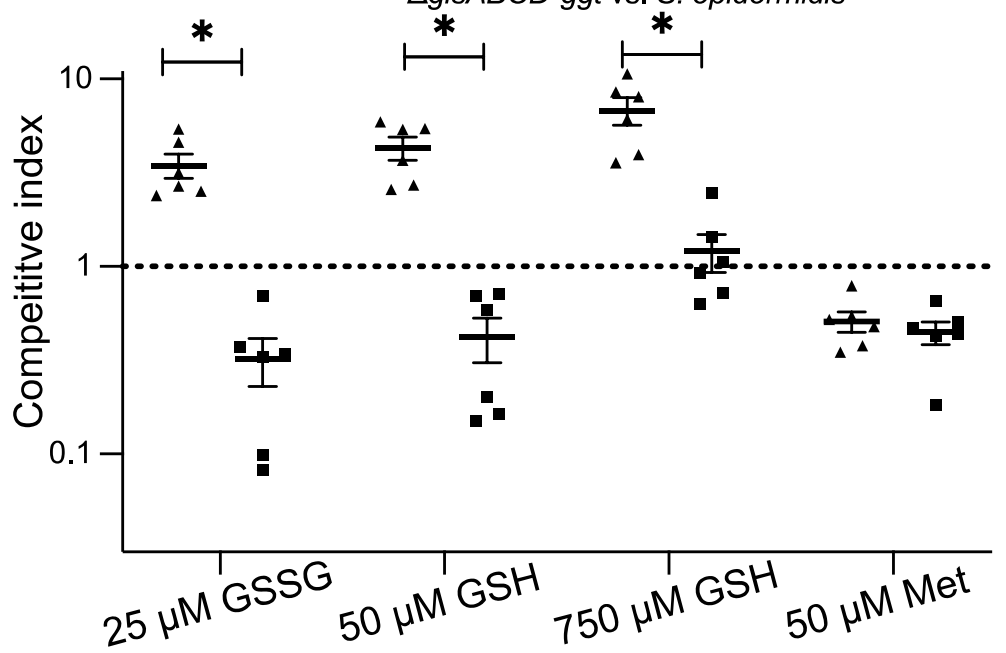

Figure 4. GisABCD is conserved in exclusive staphylococci and promotes competition in GSH- or GSSG-supplemented media. a, Heatmap depicting percent similarities of GisABCD-Ggt proteins across Staphylococci ( $S$. aureus Gis-Ggt proteins were used as the starting points for the homology searches). b, In vitro competition experiments between $S$. aureus and $S$. epidermidis strain RP62a. The line represents the mean competitive index for each individual trial. Error bars represent \pm 1 standard error of the mean. ${ }^{*}$ Indicates $p$-value $<0.05$ as determined by one-way ANOVA with Tukey's multiple test correction. 